2018

\title{
Human Activity Detection Patterns: a Pilot Study for Unobtrusive Discovery of Daily Working Routine
}

\author{
Hicham Rifai \\ Technological University Dublin \\ Paula Kelly \\ paula.kelly@tudublin.ie \\ Yoshiki Shoji \\ Technological University Dublin
}

See next page for additional authors

Follow this and additional works at: https://arrow.tudublin.ie/ehsiart

Part of the Medicine and Health Sciences Commons

\section{Recommended Citation}

Rifai, H., Kelly, P., Shoji, Y., Berry, D. \& Zallio, M. (2019). Human activity detection patterns: a pilot study for unobtrusive discovery of daily working routine. IHSI 2018: Intelligent Human System Integration, pp.143-148. doi:10.1007/978-3-319-73888-8_24

This Article is brought to you for free and open access by the ESHI Publications at ARROW@TU Dublin. It has been accepted for inclusion in Articles by an authorized administrator of ARROW@TU Dublin. For more information, please contact arrow.admin@tudublin.ie, aisling.coyne@tudublin.ie,gerard.connolly@tudublin.ie.

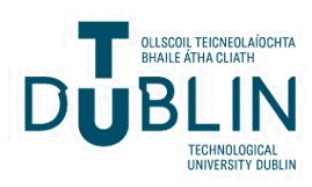


Authors

Hicham Rifai, Paula Kelly, Yoshiki Shoji, Damon Berry, and Matteo Zallio

This article is available at ARROW@TU Dublin: https://arrow.tudublin.ie/ehsiart/13 


\title{
Human activity detection patterns \\ A pilot study for unobtrusive discovery of daily working routine

\author{
Hicham Rifai ${ }^{1}$, Paula Kelly ${ }^{1}$, Yoshiki Shoji, \\ Damon Berry ${ }^{1}$, Matteo Zallio ${ }^{1}$ \\ ${ }^{1}$ Dublin Institute of Technology, Environmental Sustainability \& Health Institute, \\ t-POT Research Group, Dublin 7, Ireland \\ \{Hicham.Rifai, Paula.Kelly, Damon.Berry, Matteo.Zallio\} \\ (a) dit.ie
}

\begin{abstract}
Information technology is increasingly becoming an integral part of contemporary life. Most tasks that are performed over the course of a day, involve the use of different types of connected devices. About two billion contemporary consumers use smartphones [1]. These smartphones contain a variety of sensors that can collect information about their users such as their mobility patterns, daily activities and occupancy patterns [2]. Meanwhile other technologies such as electrical power usage are becoming increasingly "connected" through use of "smart plugs". Occupancy is an important aspect in developing responsive environments and for optimizing building performance. This work investigates the extent to which smartphones can be used to collect occupancy data in a work environment, compared to another method that uses smart power outlets for collecting occupancy data and validated against diary entries, which are recorded manually by participants each time they change their occupancy state.
\end{abstract}

Keywords: Activity Recognition · Human Factors · Human-systems Integration · Energy consumption detection · Bluetooth $\cdot$ User-centered Design $\cdot$ Privacy - Assistive Technology

\section{$1 \quad$ Introduction}

Human occupancy and presence are the main factors that cause uncertainty in building performances. This is mainly due to the complexity of human behaviour that is affected by many measurable and non-measurable factors such as age, abilities, habits, and culture. Therefore, understanding human occupancy and presence patterns would enable better management of indoor spaces by adapting the indoor space parameters based on the occupancy pattern of individuals [3]. Human activity recognition and presence detection have classically been part of computer vision and motion-sensing research [4]. However, computer vision is not only intrusive but also relatively expensive, as multiple cameras have to be installed in different locations in the environment. The main aim of the research is to verify if the use of unobtrusive, low-cost technologies 
and an analytical model can identify and recognize daily occupancy for a defined number of voluntary users working at their workstations in an open office space.

The research outcomes concern the creation of a validation protocol to be tested in a pilot study which involves the use of multiple technologies as Belkin Wemo Smart Plugs for electricity consumption monitoring, a gateway for detecting users' smartphone Bluetooth signals and a user-based diary form, used to validate the results generated by the system.

\section{Related works}

The literature points out several methods for collecting occupancy data in office environments. In Sun et al. work [6], the database associated with magnetic employee cards, used to access a building, was used to collect overtime occupancy information. In Mahdavi, et al. work [7], motion sensors were placed on the ceiling of office spaces and configured to detect motion every 100 seconds. In Andersen, et al. work [8], occupancy of workspaces was deduced from the electrical lighting ballast status in individual workspaces. In Candanedo, et al, work [9] video cameras were used to capture images of a shared office environment to determine occupancy in addition to temperature, humidity, and $\mathrm{CO} 2$ sensors. In Balaji et al. and Christensen et al. [10,11], a Wi-Fi enabled device was used to detect Wi-Fi activity and so determine the occupancy in an office space. Smartphone sensors have been used in Rana et al. work [12] to determine occupancy, mainly through the use of data from accelerometers and microphones. Lam et. al [13] used a similar approach to the one proposed here, where smart wireless outlets were placed at the workstations of employees to collect the electricity consumption at five minute intervals. In addition, a computer program was used to identify activity on each workstation computer and a wearable device (Fitbit enabled Bluetooth) was used to indicate the presence of the participants.

\section{$3 \quad$ Methodology}

Although smart plugs can influence the dynamic behavior of electrical current, it is possible to detect the approximate electrical consumption of different workstations on a second by second basis, by using devices such as Belkin Wemo Smart Plugs [14]. The proposed infrastructure for this work is composed of a set of four Belkin Wemo Smart Plugs, and an embedded Linux gateway, which collects Bluetooth information from participant's personal smartphones. This can be compared to a user-based presence register, which is manually filled in by each user.

The first step in using this infrastructure focused on developing a plan of the test workspace environment, showing the position of users' workstations, desks, chairs and any possible obstacles that would obstruct the signals. Before setting up the test area, the operation of the system at different positions of the gateway was tested. With the help of the plan, the Bluetooth proximity of the participants' mobile devices was measured and recorded in order to refine the reading thresholds for occupancy/non-occupancy of the workspace. The same process of calibration and presetting was undertaken 
for measuring the potential correct thresholds (computer in use/computer not in use) to be measured from the smart plugs.

\subsection{Calibration and data collection}

The calibration consisted of measuring the Bluetooth RSSI signal, at various distances from the gateway. It was noticed that if the smartphone was situated between 1 and 3 meters from the gateway, the RSSI signal strength was $0 \mathrm{dBm}$ in most of the measurements. The signal strength decreased gradually as the smartphone moved away from the gateway with higher measurement variation as shown in Figure 1. The box plot in the bottom right corner of Figure 1 shows measurements of the signal from phones that are situated outside the office environment which were at a distance of 12 meters from the gateway and separated by a door/wall. The maximum signal strength, outside of the room was recorded at $-19 \mathrm{dBm}$ while the minimum signal measured inside the room was $-15 \mathrm{dBm}$. After a number of tests, the mean value for the "occupancy threshold" for RSSI signal strength was chosen to be $-17 \mathrm{dBm}$. This value was then used to distinguish whether a participant was considered to be inside or outside the working environment.

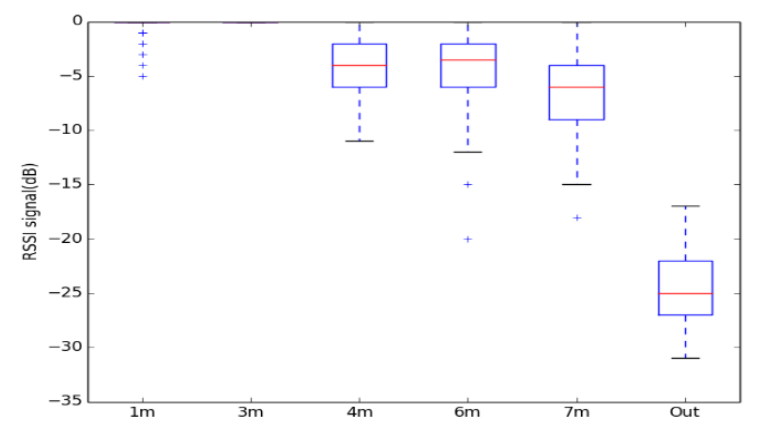

Fig. 1. Box plot of RSSI signal measurements at various distances from the gateway.

Each smart plug was connected via a Wi-Fi network with the gateway which was also used for collecting the Bluetooth signal and so acted as a receiver of both Wi-Fi and Bluetooth signals. The calibration process for the smart plug system consisted of analyzing different values, taken from smart plugs attached to each participant's workstation (measuring the combined electrical consumption at 30 seconds intervals of the desktop and monitor), by using a purpose-built Linux application utilizing the Ouimeaux Python library [15]. The next step involved the determination of the current threshold that indicates that a workstation is in use, is in standby mode or off. Figure 2 shows how the consumption drops from $27 \mathrm{~W}$ to $16 \mathrm{~W}$ when the screen switches to standby mode, but the computer is still on. A much more obvious drop in computer power consumption from $16 \mathrm{~W}$ to about $1.5 \mathrm{~W}$ can also be observed when the computer switches into sleep mode and the monitor is in standby mode. According to the collected 
data, there was a "spike" of electrical current every time a change of mode occurred. It was calculated that the screen consumes about 11-13 W while the desktop computer consumes a minimum of about $16 \mathrm{~W}$. Therefore, a jump of an amount of 11-13 W would indicate a change of state related to the participant interacting with the workstation. Prior to the start of the data collection, participants were asked to provide their Bluetooth ID and to enable Bluetooth visibility on their smartphone by "pairing" with the gateway. For reasons of anonymity and, each device and smart outlet was renamed as "participant 1,2,3,4".

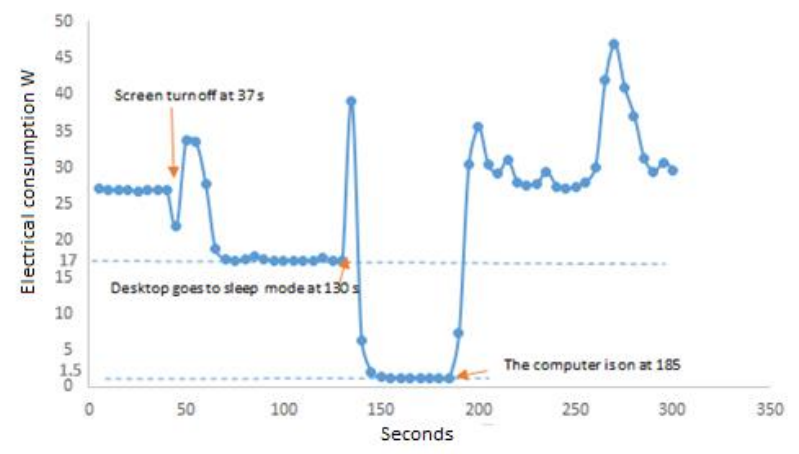

Fig. 2. Measurements of electrical consumption of screen and desktop during screen standby mode and desktop sleep mode.

\section{$4 \quad$ Findings and data evaluation}

For each participant, occupancy data were collected every 30 seconds for five days between 10:30 and 18:00 that resulted in 4500 records from the Bluetooth signals and another 4500 for power consumption, both sets of data were recorded into a MySQL database. Using the collected data, precision and recall figures were calculated using equation 1, where $\mathrm{O} / \mathrm{O}$ refers to the occupancy collection mechanism indicating an "occupant" state and the corresponding diary entry also indicates the same state, O/NO indicates that the occupancy collection mechanism indicates an "occupant" state while the corresponding diary filled by the participant indicates the opposite. Finally, NO/O refers to the occupancy collection mechanism indicating a "Non-Occupant" state while the diary indicates an "Occupant" state.

$$
\begin{gathered}
\text { Precision }=\frac{O / O}{O / O+O / N O}, \text { Recall }=\frac{O / O}{O / O+N O / O} . \\
F-\text { score }=2 \times \text { Recall } \times \frac{\text { Precision }}{\text { Precision }+ \text { Recall }}
\end{gathered}
$$


The latter method indicated occupancy, while the participant was not at the workstation $(\mathrm{O} / \mathrm{NO})$ since the monitor does not go to sleep mode immediately after the participant leaves the work station. In addition, when it happens that the participant is at the desk but he/she is not using the computer, then the system indicates no occupancy as the monitor is off $(\mathrm{NO} / \mathrm{O})$ (Table 1$)$.

Table 1. Comparison of Bluetooth and electrical consumption readings.

\begin{tabular}{|c|c|c|c|c|c|c|c|}
\hline$\#$ & Method used & $\mathbf{O} / \mathbf{O}$ & O/NO & $\mathrm{NO} / \mathrm{O}$ & Precision & Recall & F-score \\
\hline \multirow[b]{2}{*}{1} & Bluetooth based & 1479 & 14 & 24 & 0.99 & 0.98 & 0.99 \\
\hline & Power based & 1275 & 277 & 63 & 0.82 & 0.94 & 0.88 \\
\hline \multirow[b]{2}{*}{2} & Bluetooth based & 933 & 22 & 71 & 0.97 & 0.92 & 0.92 \\
\hline & Power based & 1060 & 212 & 89 & 0.83 & 0.92 & 0.87 \\
\hline \multirow[b]{2}{*}{3} & Bluetooth based & 1537 & 3 & 75 & 0.99 & 0.95 & 0.97 \\
\hline & Power based & 1324 & 211 & 95 & 0.86 & 0.93 & 0.89 \\
\hline 4 & $\begin{array}{l}\text { Bluetooth based } \\
\text { Power-based }\end{array}$ & 1574 & 99 & 197 & 0.94 & 0.88 & 0.91 \\
\hline
\end{tabular}

Figure 3 shows the behavior of electrical consumption more clearly. The smartphone-based system demonstrated the accuracy for all the participants, even if one of the four participants was located $8 \mathrm{~m}$ away from the gateway so sometimes the signal might have indicated smaller values than the threshold.
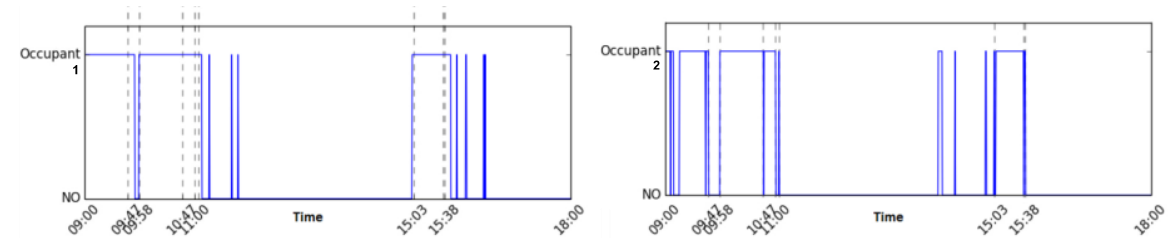

Fig. 3. Electrical consumption related to different occupants.

\section{Conclusions \& future work}

Based on the findings, we can state that the change in the level of the electrical current can easily be used to understand room occupancy at a commercial workstation, albeit with a latency of 5 to 10 minutes. On the other hand, a Bluetooth signal from a smartphone is more accurate and can validate the changing of state in room occupancy without requiring configuration of when a monitor goes to sleep. One limitation of this study concerns the recent versions of Android and iOS, which restrict the use of discovery mode for Bluetooth. The other limitation relates to the use of multiple smart outlets that communicate data over $\mathrm{Wi}-\mathrm{Fi}$ to a single gateway, which proved to be an 
unreliable approach, as some data records were lost. In perspective, this work will be extended to include a probability predictive model based on historical data and will potentially predict the probability of occupancy and deduce the most probable activity of the participant. The data collected can be condensed into a model based on the paradigm of web-based automator services that allows users to create chains of simple conditional statements and managed by a probabilistic/mathematical framework for modelling decision making [16].

\section{References:}

1. Andrews, S., Ellis, D., Shaw, H., Piwek, L.: Beyond Self-Report: Tools to Compare Estimated and Real-World Smartphone Use. PLOS ONE. 10, e0139004 (2015).

2. Harari, G., Lane, N., Wang, R., Crosier, B., Campbell, A., Gosling, S.: Using Smartphones to Collect Behavioral Data in Psychological Science. Perspectives on Psychological Science. 11, 838-854 (2016).

3. Gunay, H., O'Brien, W., Beausoleil-Morrison, I.: A critical review of observation studies, modeling, and simulation of adaptive occupant behaviors in offices. Building and Environment. 70, 31-47 (2013).

4. 4.Liu, L., Peng, Y., Wang, S., Liu, M., Huang, Z.: Complex activity recognition using time series pattern dictionary learned from ubiquitous sensors. Information Sciences. 340-341, 41-57 (2016).

5. Liu, L., Peng, Y., Wang, S., Liu, M., Huang, Z.: Complex activity recognition using time series pattern dictionary learned from ubiquitous sensors. Information Sciences. 340-341, 41-57 (2016).

6. Sun, K., Yan, D., Hong, T., Guo, S.: Stochastic modeling of overtime occupancy and its application in building energy simulation and calibration. Building and Environment. 79, 112 (2014).

7. Mahdavi, A., Tahmasebi, F.: Predicting people's presence in buildings: An empirically based model performance analysis. Energy and Buildings. 86, 349-355 (2015).

8. Andersen, P., Iversen, A., Madsen, H., Rode, C.: Dynamic modeling of presence of occupants using inhomogeneous Markov chains. Energy and Buildings. 69, 213-223 (2014).

9. Andanedo, L., Feldheim, V.: Accurate occupancy detection of an office room from light, temperature, humidity and $\mathrm{CO} 2$ measurements using statistical learning models. Energy and Buildings. 112, 28-39 (2016).

10. Balaji, B., Xu, J., Nwokafor, A., Gupta, R., Yuvraj Agarwal, Y.: Sentinel: occupancy based HVAC actuation using existing WiFi infrastructure within commercial buildings. In Proceedings of the 11th ACM Conference on Embedded Networked Sensor Systems. (2013).

11. Christensen, K., Melfi, R., Nordman, B., Rosenblum, B., Viera, R.: Using existing network infrastructure to estimate building occupancy and control plugged-in devices in user workspaces. International Journal of Communication Networks and Distributed Systems. 12, 4 (2014).

12. Rana, R., Kusy, B., Wall, J., Hu, W.: Novel activity classification and occupancy estimation methods for intelligent HVAC (heating, ventilation and air conditioning) systems. Energy. 93, 245-255 (2015).

13. Lam, K., Zhao, J., Wirick, J., Qi, M.: An EnergyPlus whole building energy model calibration method for office buildings using occupant behavior data. 2014 ASHRAE/IBPSA-USA Building Simulation Conference. 160-167 (2014).

14. Jason., C.: Review: Belkin WeMo Switch and Motion. (2013). 
15. Pradeep, L., Ambati, R., Irwin, D.: AutoPlug: An automated metadata service for smart outlets. Green and Sustainable Computing Conference, IEEE. 1-8 (2016).

16. Zallio M., McGrory J., Berry D., 2017. IoT Based Recipes for Enabling Senior Citizens: Stakeholders Views on How Integration of IoT and Web Services Can Enhance Well-Being and Inclusion of Older People. Design for Inclusion, Chapter No: 7, Springer International, proceedings of the AHFE 2017, July 17-21, 2017, Los Angeles, CA, USA. 